\title{
A case of Noonan syndrome with skull defect due to vitamin $\mathrm{D}$ deficiency rickets
}

\author{
Syunsuke Nagara ${ }^{1}$, Shinji Usui ${ }^{1}$, Miwa Kawashiri ${ }^{1}$, Masashi Kondo ${ }^{2}$, and Atsushi Yamagishi ${ }^{1}$ \\ ${ }^{1}$ Department of Pediatrics, Japanese Red Cross Takayama Hospital, Gifu, Japan \\ ${ }^{2}$ Department of Neonatology, Gifu Prefectural General Medical Center, Gifu, Japan
}

\begin{abstract}
We report the case of a boy with partial skull defects in addition to widespread craniotabes due to vitamin D deficiency rickets. He was born at 30 wk and $4 \mathrm{~d}$ of gestation (birth weight, $2406 \mathrm{~g}$ ). At $77 \mathrm{~d}$ of age, clinical examination of the head revealed widespread craniotabes of the occipital region centered around the lambda suture, and palpation revealed a defect of about $1 \mathrm{~cm}$ in the parietal bone of the left occipital region. Cranial computed tomography showed thinning of the cortex and bone defects in the parietal bones bilaterally, as well as in the left occipital bone. At 3 mo of age, he was diagnosed with vitamin D deficiency rickets and was administered alfacalcidol for 4 mo. Although patients with vitamin $\mathrm{D}$ deficiency rickets are prone to fractures, bone defects, as in this case, have not been reported. In addition to vitamin $\mathrm{D}$ deficiency rickets, the causes of the bone defects, in this case, are hypothesized to be abnormalities in the Ras-mitogen activated protein kinase pathway associated with Noonan syndrome, and long-term compression of the back of the head. However, there are no other similar reports, and further ones need to be accumulated.
\end{abstract}

Key words: Noonan syndrome, vitamin D deficiency rickets, skull defect, craniotabes

\section{Introduction}

Noonan syndrome (MIM163950) is an autosomal dominant developmental disorder characterized by short stature, facial dysmorphism, cardiovascular defects, and skeletal and hematological abnormalities (1). Hyperactive mutations of molecules in the Ras-mitogen activated protein kinase (MAPK) pathway, particularly PTPN11, have been found in Noonan syndrome, and the identification of these mutations has aided in elucidating the pathology of this syndrome (2). Skeletal disease craniosynostosis, but not bone defects, has recently been reported as a complication of Noonan syndrome.

Craniotabes is a physical finding in newborn infants detected by careful palpation of the infant's head. It represents an area of softened cranial bone. If the patient's history and examination findings are normal, no further investigations are required. However, if craniotabes are found on the posterolateral portions of the skull, in contrast to the vertex location of benign craniotabes, further investigations are required, and the differential diagnosis includes vitamin $\mathrm{D}$ deficiency rickets (3). We report a case of a child with Noonan syndrome complicated by vitamin $\mathrm{D}$ deficiency rickets with widespread craniotabes.

\section{Case Report}

A 27-yr-old pregnant woman presented with polyhydramnios (amniotic fluid index, $42.4 \mathrm{~cm}$ ) and fetal hydrops at $30 \mathrm{wk}$ and $0 \mathrm{~d}$ of gestation. She was transferred to a higher-level medical facility on the same day, and a cesarean section was performed at $30 \mathrm{wk}$ and $4 \mathrm{~d}$ of gestation. She delivered a male infant with a birth weight of $2406 \mathrm{~g}$. The Apgar scores were 1 and 6 at 1 and 5 min, respectively. The neonate experienced respiratory distress syndrome, and an artificial lung surfactant was administered after endotracheal intubation, and ventilator management was commenced. A pleural cavity drain was placed for bilateral pleural effusions at $4 \mathrm{~d}$ of age, and continuous pleural effusion drainage was started. Enteral nutrition of medium-chain triglyceride milk was commenced along with intravenous octreotide. Prednisolone was administered, and the pleural effusion resolved at $23 \mathrm{~d}$ of age. The pleural drains were removed at $28 \mathrm{~d}$ of age, and octreotide and prednisolone were stopped sequentially. The infant was extubated at 32 $\mathrm{d}$ of age. Breastfeeding was commenced at $36 \mathrm{~d}$ of age,

Received: August 5, 2020 Accepted: October 23, 2020

Corresponding author: Atsushi Yamagishi, M. D., Department of Pediatrics, Japanese Red Cross Takayama Hospital, 3-11 Tenmanchou, Takayama, Gifu 506-8550, Japan

E-mail: yamagishi_a@takayama.jrc.or.jp 


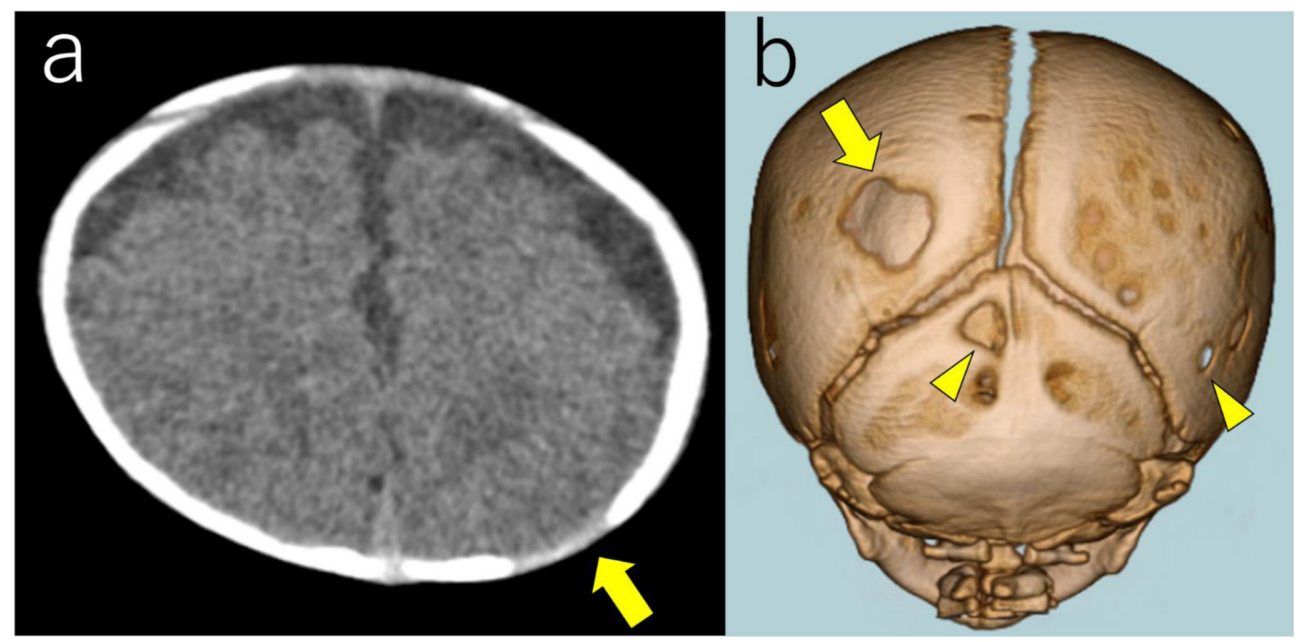

Fig. 1. Computed tomography of the infant's head at $77 \mathrm{~d}$. The arrows indicate the same bone defect site in the axial cross-sectional image (a) and three-dimensional reconstructed image (b). The arrowhead points to the other bone defects (a).

and the infant was transferred to our hospital at $47 \mathrm{~d}$ of age. During prior hospitalization, no abnormalities in alkaline phosphatase (ALP), calcium, or phosphate levels were observed [ALP, 1188 IU/L (47 d of age)]. There were no data on X-ray findings and 25-hydroxyvitamin $\mathrm{D}(25(\mathrm{OH}) \mathrm{D})$ level from the previous hospital.

After admission to our hospital at $50 \mathrm{~d}$ of age, breast milk was changed to an anti-regurgitation formula due to poor oxygenation associated with gastroesophageal reflux disease. Although no abnormalities of the head were noticed at the time of admission, clinical examination of the head at $77 \mathrm{~d}$ of age revealed widespread craniotabes of the occipital region centered around the lambda suture, and palpation revealed a defect of about $1 \mathrm{~cm}$ in the parietal bone of the left occipital region. Cranial computed tomography showed thinning of the cortex and bone defects in the parietal bones bilaterally, as well as in the left occipital bone (Fig. 1). There were no abnormal findings in the skull defect site on magnetic resonance imaging of the head, and the patient was discharged at $80 \mathrm{~d}$ of age.

Noonan syndrome is a differential diagnosis based on the course of fetal hydrops, hearing loss, and congenital pulmonary valvular stenosis. Physical examination did not reveal any of the morphological features of Noonan syndrome other than interocular dissociation and low auricles. Juvenile myelomonocytic leukemia and Noonan syndrome-related myeloproliferative disorder were listed as differential diagnoses as the infant received repeated blood transfusions during his hospitalization due to anemia of monocytosis. As the treatment of these two diseases is different, genetic and bone marrow tests were performed post-discharge. A genetic test, performed with parental consent, revealed a missense heterozygous mutation (c.188A > G[p.Y63C]) of the PTPN11 gene, which has previously been reported as a pathogenic mutation in Noonan syndrome (4), and Noonan syndrome was diagnosed. He was subsequently diagnosed with
Noonan syndrome-related myeloproliferative disorder, and the anemia improved without intervention.

At 3 mo of age, radiography of the wrist joint showed slight cupping of the ulna end, and blood examination revealed that the $25(\mathrm{OH}) \mathrm{D}$ level was $<4.0 \mathrm{ng} / \mathrm{mL}$. The intact parathyroid hormone level was $136 \mathrm{pg} / \mathrm{mL}$ (reference value, 15-65 pg/mL), the ALP level was 1517 IU/L (hyperphosphatasemia, > 1200 IU/L), the calcium level was $9.1 \mathrm{mg} / \mathrm{dL}$ (reference value, $9.4-10.8 \mathrm{mg} / \mathrm{dL}$ ), the albumin level was $3.7 \mathrm{~g} / \mathrm{dL}$ (reference value, 3.2-4.3 $\mathrm{g} / \mathrm{dL}$ ), and the inorganic phosphate level was $6.1 \mathrm{mg} / \mathrm{dL}$ (reference value, $5.3-10.3 \mathrm{mg} / \mathrm{dL}$ ). These findings led to a diagnosis of vitamin $\mathrm{D}$-deficient rickets, and the patient was administered alfacalcidol at a daily dose of $0.1 \mathrm{\mu g} /$ $\mathrm{kg}$. We did not supplement native vitamin $\mathrm{D}$ and did not provide lifestyle counseling for the mother to encourage more sunlight exposure. Despite treatment, at 4 months of age, the ALP level increased to 2122 IU/L before returning to the normal range at 5 months of age. The serum $25(\mathrm{OH}) \mathrm{D}$ level increased to $31.8 \mathrm{ng} / \mathrm{mL}$ at $6 \mathrm{mo}$ of age. The thinned part of the bone cortex had hardened into the bone at 6 months of age, and the bone defects disappeared on computed tomography at 7 mo of age. Oral administration of alfacalcidol was discontinued. At both the previous hospital and our hospital, the ALP level was estimated using the method recommended by the Japan Society of Clinical Chemistry. The maternal $25(\mathrm{OH}) \mathrm{D}$ level was not estimated.

\section{Discussion}

Of interest, in this case, is the presence of partial skull defects in addition to widespread craniotabes due to vitamin $\mathrm{D}$ deficiency rickets. Although patients with vitamin $\mathrm{D}$ deficiency rickets are prone to fractures, bone defects, as in this case, have not been reported. In combination with vitamin $\mathrm{D}$ deficiency rickets, the bone defect, in this case, is speculated to be due 
to abnormalities in the Ras-MAPK signaling pathway found in Noonan syndrome, as well as the long-term compression of the back of the head.

Dysregulation of the extracellular signal-regulated kinase (ERK) MAPK pathway is associated with human skeletal abnormalities, including Noonan syndrome. Deletion of the kinases upstream of ERK/ MAPK has been shown to result in severe osteopenia and cleidocranial dysplasia, similar to that seen in both humans and mice with impaired runt-related transcription factor 2 function (5). Although there are no reports of cleidocranial dysplasia associated with Noonan syndrome, disruption of skeletal homeostasis due to an abnormal MAPK pathway may be related to the bone defect in this case.

Although the etiology of craniotabes is unclear, long-term head compression is known to be one of its causes and may result in temporarily delayed and localized calvarial ossification (6). In this case, ventilator management in the supine position was performed from birth to 32 days of age, resulting in a prolonged load on the skull and delay in the calcification of the osteoid. It is also speculated that calcification of the skull was delayed due to the onset of rickets, thereby leading to bone defects.
There are a few limitations to this case report. First, the exact role of the Ras-MAPK pathway in the regulation of bone homeostasis is not clear, and the association between PTPN11 mutations and bone defects has not yet been confirmed. Second, there is no evidence that the cause of the bone defect, in this case, is a combination of Noonan syndrome, long-term bed rest, and vitamin $\mathrm{D}$ deficiency rickets. To the best of our knowledge, this is the first report of Noonan syndrome with skull defects and vitamin $\mathrm{D}$ deficiency rickets, and more similar cases need to be accumulated.

Consent: Informed consent was obtained from the parents of the patient for publication of this case report and the accompanying images.

Conflict of interests: The authors declare no conflict of interest.

\section{Acknowledgments}

We are grateful to Dr. Norifumi Yokoyama (Gifu Municipal Hospital) and Dr. Hideki Muramatsu (Nagoya University Hospital) for their help.

\section{References}

1. Tartaglia M, Kalidas K, Shaw A, Song X, Musat DL, van der Burgt I, et al. PTPN11 mutations in Noonan syndrome: molecular spectrum, genotype-phenotype correlation, and phenotypic heterogeneity. Am J Hum Genet 2002;70: 1555-63. [Medline] [CrossRef]

2. Tartaglia M, Martinelli S, Stella L, Bocchinfuso G, Flex E, Cordeddu V, et al. Diversity and functional consequences of germline and somatic PTPN11 mutations in human disease. Am J Hum Genet 2006;78: 279-90. [Medline] [CrossRef]

3. Fox GN, Maier MK. Neonatal craniotabes. Am Fam Physician 1984;30: 149-51. [Medline]

4. Tartaglia M, Mehler EL, Goldberg R, Zampino G, Brunner HG, Kremer H, et al. Mutations in PTPN11, encoding the protein tyrosine phosphatase SHP-2, cause Noonan syndrome. Nat Genet 2001;29: 465-8. [Medline] [CrossRef]

5. Kim JM, Yang YS, Park KH, Oh H, Greenblatt MB, Shim JH. The ERK MAPK pathway is essential for skeletal development and homeostasis. Int J Mol Sci 2019;20: 1803. [Medline] [CrossRef]

6. Graham JM. Smith's recognizable patterns of human deformation. 3rd ed. Amsterdam: Elsevier; 2007, p.226-9. 